\title{
Identification of dysfunctional human-dog dyads through dog ownership histories
}

\author{
Rute Canejo-Teixeira ${ }^{1, *}$, Isabel Neto ${ }^{1}$, Luís V. Baptista ${ }^{2}$ and Maria Manuela Rodeia Espada Niza \\ ${ }^{1}$ CIISA, Faculdade de Medicina Veterinária/Ulisboa, Lisbon, Portugal \\ ${ }^{2}$ Faculdade de Ciências Sociais e Humanas, Universidade Nova de Lisboa, Lisbon, Portugal
}

\begin{abstract}
The human-dog relationship is at least 16,000-years old and is mutually beneficial to both dyadic members. When the human-dog relationship becomes dysfunctional, however, there can be serious consequences for both parties and for society. Unfortunately, dysfunctional dyads are normally only identified after consequences have been felt (e.g., dog-human aggression) limiting the action that can be taken to prevent such occurrences. To evaluate whether these dysfunctional dyads can be preemptively identified, a questionnaire analyzing the owners' dog health care histories was administered to an urban dog owning population. Multiple correspondence analysis $(n=1,385)$ was conducted and identified three clusters accounting for $37.1 \%$ of the total variance, while four moderate positive correlations were found: "unspecified trauma" with "vehicular trauma" $(r=0.303, p<0.001)$, "bitten" with "bit other animal" $(r=0.345$, $p<0.001)$, "bit a person" with "bit other animal" $(r=0.369, p<0.001)$, and "chronic illness" with "hospitalized" $(r=0.297, p<0.001)$. These results suggest that a simple questionnaire can identify potential characteristics of functional and dysfunctional dyads. In functional dyads, humans tend to be responsible for their dogs' well-being, while dysfunctional dyads show the opposite characteristics, reporting experience with trauma and dog aggression.
\end{abstract}

Keywords: Dysfunctional dyads, Human-dog bond, MCA, Ownership characteristics, Questionnaire.

\section{Introduction}

The human-dog relationship is believed to be at least 16,000-years old and to have evolved due to its mutually beneficial nature (Wang et al., 2015). Within a functional human-dog dyad, the human partner benefits in a variety of ways, from using dog's capacity to work (Sanders, 2000; Greatbatch et al., 2015) through to its value as a companion animal (Davis et al., 2004; Wood et al., 2005; Nimer and Lundahl, 2007; Kuban et al., 2016), while the dog has its basic needs provided for (food, shelter, veterinary care, etc.). In these dyads, the human is generally considered to be the responsible party (Houpt et al., 2007), and as such must insure that the relationship is beneficial not only to its' two members, but to society at large (Wood et al., 2005). However, when these human-dog dyads become dysfunctional, they can present a risk not only to each member but to the general public (Lambert et al., 2015; Mongillo et al., 2015). These dysfunctional human-dog relationships have received much attention in the recent years (O'Haire, 2010; Rehn and Keeling, 2016) in order to understand their origins and attempt to correct or at least minimize their effects.

One of the hallmarks of dysfunctional human-dog dyads is the tendency for the dog to develop problem behaviors (Bennett and Rohlf, 2007; Meyer and Forkman, 2014), the most obvious of which is dog on human aggression (Fatjo et al., 2007). Dogs in dysfunctional dyads are often allowed to roam
(Mustiana et al., 2015), making them more likely to become involved in a vehicular accident, to harm other animals and to cause the destruction of the property. When such situations occur, the solution found for these problem dogs is frequently euthanasia (Galvis et al., 2015). Canine euthanasia is also a frequent solution sought out by the owners for their personal convenience (Overall, 2010; Coe et al., 2014). These consequences of problematic dyads can be severe for both human and dog, making the understanding of why and how they develop a matter of great importance. However, these dyads are notoriously difficult to identify and study, since the human partner is unlikely to easily volunteer personal information (Bennett and Rohlf, 2007; Rohlf et al., 2010).

Identification and study of dysfunctional dyads have mostly been conducted after the dog has manifested some kind of behavioral problem (Guy et al., 2001), frequently dog-human aggression (Keuster et al., 2006; Le Brech et al., 2016). The concern with this approach is that it takes place after the fact, making preventive measures impossible to implement. Theoretically, it should be possible to identify the quality of the humandog relationship through the knowledge of owners' dog health care histories, willingness to abide by animal welfare laws and the provision of necessary veterinary care (Rohlf et al., 2010).

The aim of this study was to evaluate whether dysfunctional human-dog dyads could be identified by 
analyzing each owner's dog health care history and by finding patterns or groupings that may occur. Through the administration of a simple yes/no questionnaire to dog owners in an urban setting, identification of general incidences that have been associated with dysfunction could be possible (Guy et al., 2001; Overall, 2010; Coe et al., 2014; Mustiana et al., 2015). To our knowledge, this is the first time that ownership history has been studied in this light and it could lead to the early detection of dysfunctional dyads. Such preemptive identification may help regulatory agencies to detect the presence of dysfunctional human-dog dyads, thereby justifying the implementation of specific preventive programs (Lakestani and Donaldson, 2015).

\section{Materials and Methods}

A simple, one-page questionnaire was developed with three distinct question categories. The first section consisted of a single question regarding the number of dogs the respondent has cared for in his or her life up until the moment they filled out the questionnaire. The second section asked binary questions regarding owner experience with different medical occurrences in their dogs' lives. The final section asked for information regarding the owner's residential parish.

As suggested by Wiseman-Orr et al. (2004), in the second section of the questionnaire, the vast gambit of possible disease processes that can occur in a dog's lifetime where condensed into simple categories. These categories used simple terms that owners would easily understand and incidents they would most likely remember (Robinson et al., 2015; 2016). Since dog health care issues that can be time consuming and/or costly are very likely to be remembered, owners were asked whether they had owned a dog that had suffered acute illness, chronic illness, unspecified, or vehicular trauma or that had been hospitalized. These five health occurrences can be common within a normal canine life span and by using simplified, non-medical terminology (by asking about chronic illness in general as opposed to renal insufficiency, for example), the owner would be more likely to correctly identify the occurrence. Situations involving dog bites, whether the dog in question is the victim or the aggressor, are very likely to be remembered since these situations can be traumatic occurrences. As such, owners were asked if their dog had been bitten, had bitten another animal or a person. It was important to assess if experience with euthanasia could be used to identify potential problem dyads, since it is not uncommon for veterinarians to come across requests for medically unjustified euthanasia (Yeates and Main, 2011). To evaluate this possibility, owners were asked if they had ever had a dog euthanized.

The questionnaire was administered first to a small sample of dog owners at the Teaching Hospital at the Faculty of Veterinary Medicine/University of Lisbon, Portugal. This test group was questioned regarding ease of understanding and clarity of the questions.
Subsequent appropriate changes were made where necessary.

Questionnaires were then distributed throughout the Greater Lisbon Metropolitan Area to various small animal hospitals, clinics, and during municipal antirabies vaccination programs for a period of $8 \mathrm{mo}$. Dog owners were asked to complete the questionnaire while in the waiting room. Care was taken in trying to include at least one clinic, hospital, or municipal kennel from each of the 18 different parish districts within the Greater Lisbon Metropolitan Area to obtain as representative a sample as possible. Questionnaires were also made available online using Google Forms ${ }^{\mathrm{TM}}$ during the same period, and its existence publicized on the Teaching Hospital at the Faculty of Veterinary Medicine/University of Lisbon website and social media.

Since the data obtained from the questionnaires was nominal in nature, an initial exploratory analysis was conducted using multiple correspondence analysis (MCA). The data were further analyzed using two-way, and where appropriate, three-way chi-square analyses. IBM SPSS Statistics for Windows, version 22.0 was used for all the statistical analysis.

\section{Results}

\section{Descriptive statistics}

A total of 1,385 questionnaires were completed at the end of the 8-mo period, $733(52.9 \%)$ online and 653 $(47.1 \%)$ at the various hospitals, clinics, and municipal anti-rabies campaigns which agreed to participate. For the first section of the questionnaire, 1,371 valid answers were obtained. All 1,385 individuals completed the middle section, and every individual had at least one medical occurrence to report. The last section obtained 1,242 valid answers and was excluded from this analysis since more than $10 \%$ of the responses obtained were invalid. When asked binary questions regarding their experience with dog health care histories, more than half of the participants reported having had at least one dog with an acute illness, chronic illness, or hospitalized (Fig. 1). Experience with aggressive behaviors was less commonly reported, and around one quarter of the participants reported experience with traumatic events (Fig. 1).

\section{Multiple correspondence analyses}

For this analysis, the two dimensions selected, which together accounted for $37.1 \%$ of the variance observed in the samples (Table 1) showed three clear clusters (Fig. 2). In line with other research, exploratory in nature (Costa et al., 2013), a Cronbach's alpha lower than 0.7 was accepted due to the heterogeneous nature of the data as well as the reduced number of questions in the questionnaire (Tavakol and Dennick, 2011).

Although none of the discrimination measures were $>0.5$, three clusters had similar discrimination measures (Table 2). The first cluster, furthest from the origin in dimension 2, groups owners reporting the 
variable "bit another animal" with the total number of dogs the individual reported having cared for in their lifetime. The second cluster, furthest from the origin in dimension 1, groups owners reporting the variables "vehicular trauma," "unspecified trauma," and "bitten." Finally, the last cluster, groups owners reporting both the variables "chronic illness" and "hospitalized." Further observation of the MCA analysis allows for the observation that the variable "euthanized" has been placed at the origin of both dimensions, suggesting that it represents the variable with the least deviation form independence in the sample.

Bivariate correlations between variables in dimension 1 were identified (transformed variables) and found to be the same as those in dimension 2. Only correlations $\geq 0.3$ were considered to be relevant (Costa et al., 2013) and as presented in Table 3 the variable "unspecified trauma" correlated significantly with "vehicular trauma," "bitten" correlated with "bit other animal," "bit a person" correlated with "bit other animal," and the variable "chronic illness" correlated with "hospitalized." It is important to note that this information arises from self-reporting data and as such correlation does not equal causation.

\section{Chi-square analysis of cluster variables}

Variables identified as having potential significant deviation from independence with MCA were further explored thought the use of chi-square analyses. The

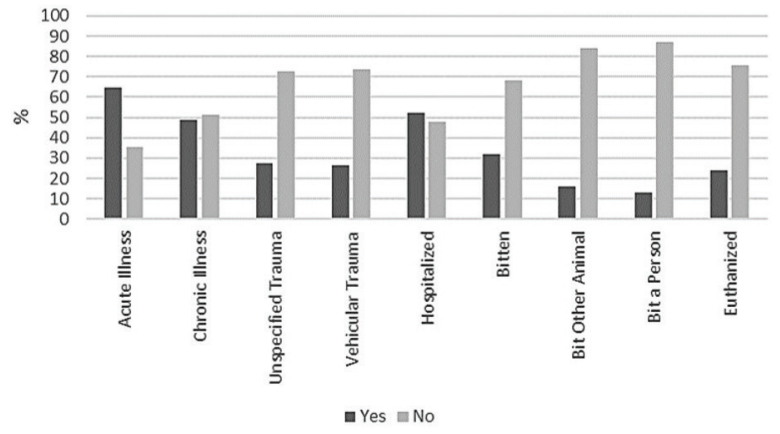

Fig. 1. Binary responses to the second section of the questionnaire regarding medical occurrences experienced while caring for one or more dogs. Medical occurrences were simplified into easy to understand occurrences more likely to be remembered by the owner.

Table 1. MCA model summary.

\begin{tabular}{cccc}
\hline \multirow{2}{*}{ Dimension } & $\begin{array}{c}\text { Cronbach's } \\
\text { alpha }\end{array}$ & \multicolumn{2}{c}{ Variance accounted fsor } \\
\cline { 3 - 4 } & 0.662 & 2.473 & 0.247 \\
(Eigen value) & Inertia \\
\hline 1 & 0.211 & 1.234 & 0.123 \\
\hline Total & & 3.706 & 0.371 \\
Mean & $0.512^{\mathrm{a}}$ & 1.853 & 0.185 \\
\hline
\end{tabular}

${ }^{a}$ Mean cronbach's alpha is based on the mean Eigen value. potential association between owners reporting chronic illness and those who reported hospitalization was significant, with $67.5 \%$ of those having experienced chronic illness with one or more dogs also referring hospitalization $\left(\chi^{2}=122.131, \mathrm{df}=1, p<0.001\right)$. In the case of owners reporting having had at least one dog suffering vehicular trauma, 50.3\% also reported unspecified trauma significantly more than expected $\left(\chi^{2}=127.310, \mathrm{df}=1\right.$, $p<0.001)$. Individuals who reported having cared for more than 11 dogs in their life-time reported having had at least one dog that bit another animal significantly more than expected $\left(\chi^{2}=85.236, \mathrm{df}=3, p<0.001\right)$. In cases where the owner reports one or more biting occurrence, $34.8 \%$ of those reporting a dog having been bitten also report more $\operatorname{dog}(\mathrm{s})$ that bit other animals $\left(\chi^{2}=164.547\right.$, $\mathrm{df}=1, p<0.001)$, and of those reporting $\operatorname{dog}(\mathrm{s})$ that have bitten a person $51.1 \%$ also cite having one or more dogs that bit other animals $\left(\chi^{2}=188.522, \mathrm{df}=1, p<0.001\right)$.

As a result of this last finding, a three-way contingency table was calculated. Although individuals who reported having cared for at least one dog which was bitten also reported having at least one dog that had bitten other animals, independently of whether or not they also reported a dog that bit a person $\left(\chi^{2}=46.578\right.$, $\mathrm{df}=1, p<0.001$ and $\chi^{2}=87.436$, $\mathrm{df}=1, p<0.001$, respectively), the association is much stronger within the population that reported having had at least one dog that bit a person (Cramer's $V=0.506, p<0.001$ versus Cramer's $V=0.270, p<0.001)$.

Analysis of the second cluster identified by MCA showed that the individuals who had not cared for dogs that suffered unspecified trauma also report less

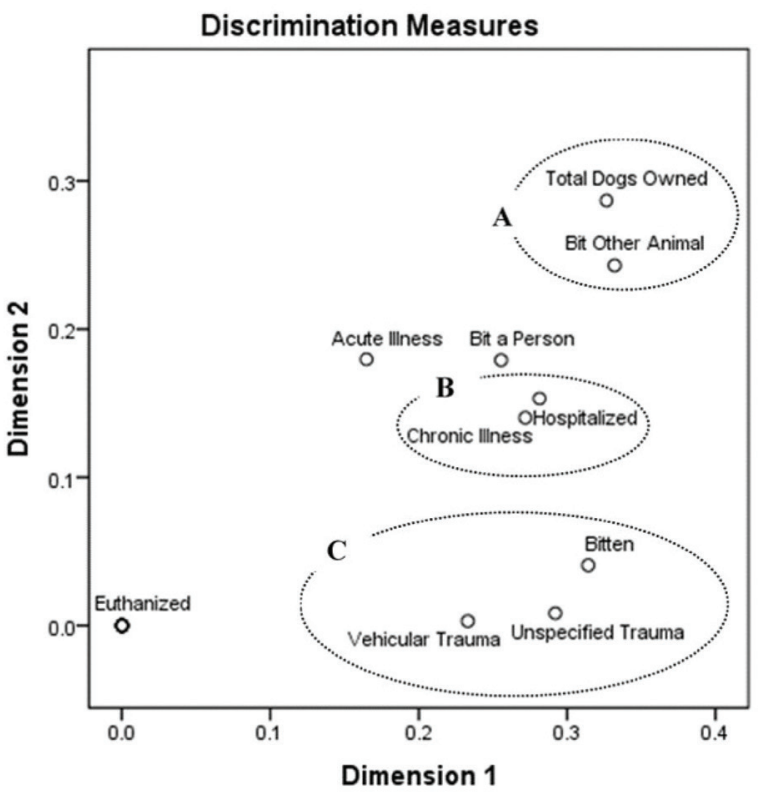

Fig. 2. MCA dimensions. Three clear groupings have been circled; (A) total dogs owned and bit other animal, (B) hospitalized and chronic illness, (C) vehicular trauma, unspecified trauma and bitten. 
Table 2. MCA dimensions discrimination measures.

\begin{tabular}{lccc}
\hline \multirow{2}{*}{ Dogs } & \multicolumn{2}{c}{ Dimension } & \multirow{2}{*}{ Mean } \\
\cline { 2 - 3 } & $\mathbf{1}$ & $\mathbf{2}$ & \\
\hline Total dogs owned & 0.327 & 0.287 & 0.307 \\
Acute illness & 0.165 & 0.180 & 0.172 \\
Chronic illness & 0.272 & 0.140 & 0.206 \\
Unspecified trauma & 0.292 & 0.008 & 0.150 \\
Vehicular trauma & 0.233 & 0.003 & 0.118 \\
Bitten & 0.314 & 0.041 & 0.178 \\
Hospitalized & 0.281 & 0.153 & 0.217 \\
Bit other animal & 0.332 & 0.243 & 0.288 \\
Bit a person & 0.256 & 0.179 & 0.217 \\
Euthanized & 0.000 & 0.000 & 0.000 \\
Active total & 2.473 & 1.234 & 1.853 \\
\hline
\end{tabular}

experience with dogs bitten and suffering vehicular trauma $\left(\chi^{2}=21.445, \mathrm{df}=1, p<0.001\right)$.

It is interesting to note that in the sample of dog owners in study, there was no single or group of medical occurrences that would make an individual more likely to choose to euthanize their dog or dogs.

\section{Discussion}

When the relationship between human and dog works well, the two individuals form a functional human-dog dyad that has been shown to be mutually beneficial (O'Haire, 2010; Wang et al., 2015). It is when these dyads become dysfunctional that they can pose a risk to humans, animals, and the community (Lambert et al., 2015; Mongillo et al., 2015). The most studied consequence of dysfunction is aggressive canine behavior, namely, dog bites (Fatjo et al., 2007). However, inappropriate canine aggression is not the only sign of potential dysfunction, nor is it the only one that presents a clear and present danger to all the parties involved. Dogs that are not provided with adequate veterinary care can represent a risk to public health (Lambert et al., 2015; Sterneberg-van der Maaten et al., 2016), those that are allowed to roam present a clear risk to public safety (Mustiana et al., 2015) and dogs that develop behavior problems are at risk of euthanasia (Bower, 2014), abandonment (Diesel et al., 2010), and can be difficult to re-home (Coe et al., 2014). Before strategies to correct these issues can be developed and implemented, the human-dog dyad requires further study to understand the underlying causes of dysfunction (Meyer and Forkman, 2014). One of the main challenges to dyadic research is persuading the human partner at the core of a dysfunctional dyad to participate in studies that require the provision of personal information (Bennett and Rohlf, 2007; Rohlf et al., 2010). These owners may feel that such information could bring into question their moral and

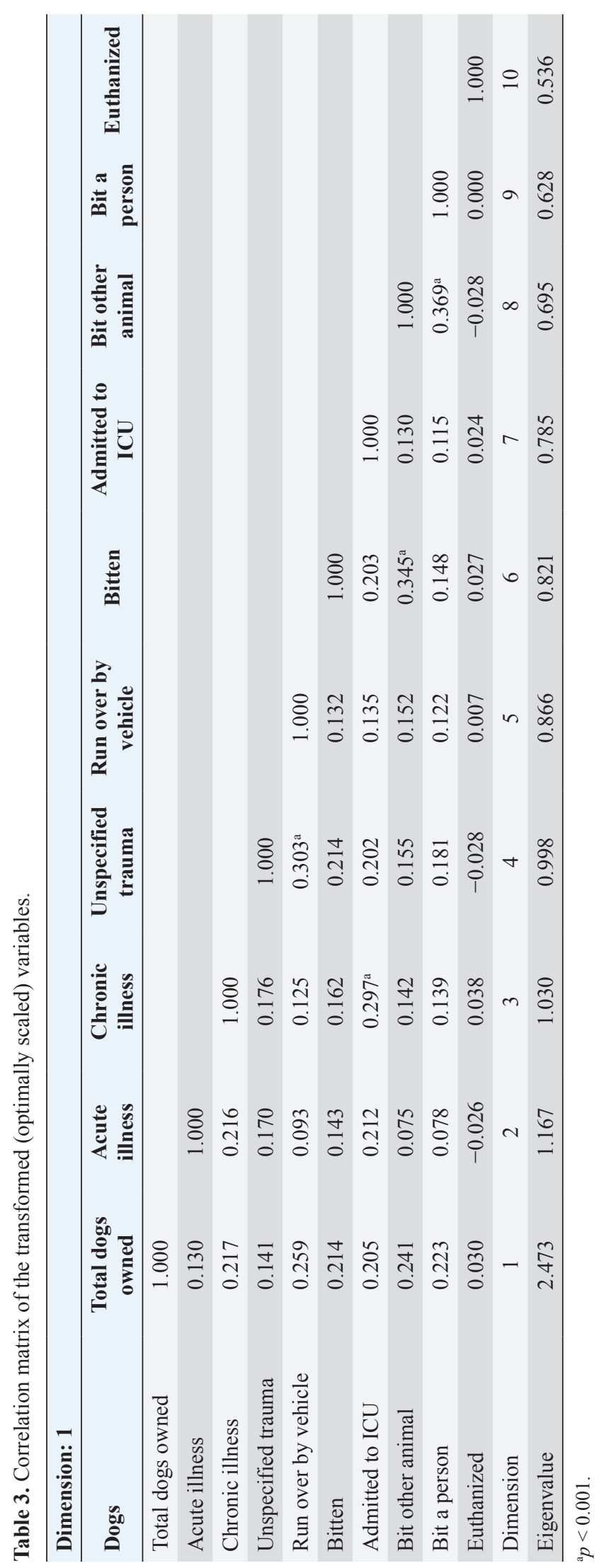


ethical principles, that they will be judged negatively in other aspects of their lives, or they may be reluctant to have their fears of poor dog ownership confirmed. Taking all these facts into consideration, a different approach was implemented. This study aimed to evaluate dog health care histories (chronic disease, trauma, and euthanasia) with the intent of find patterns within human-dog dyads that may contribute to the identification of dysfunctional in a preemptive way.

It has been suggested that the most visible sign of functional human-dog dyads, especially to veterinary professionals, is the willingness of an owner, as caregiver, to provide adequate medical care to their dog (Mariti et al., 2012; Rohlf et al., 2012). To assess this issue, the questionnaire focused on diseases and hospitalization of each individual dog, as opposed to asking questions regarding willingness that owners may find too personal or invasive.

In this study, $67.5 \%$ of owners who reported having had a dog that suffered from a chronic illness also reported significantly more experience with hospitalization. Most chronic illnesses in dogs require some period of hospitalization during the disease process (Polzin, 2013; Pouchelon et al., 2015). So, it makes sense that these owners could represent functional human-dog dyads, since they are conscientious of their responsibility to provide adequate medical care. This may not be the case with less motivated owners, such as those more likely to be a part of dysfunctional dyads (Bennett and Rohlf, 2007; Rohlf et al., 2010).

In this population, $77.7 \%$ of owners who did not report experience with a dog suffering unspecified trauma also failed to report experience with a dog victim of vehicular trauma or being bitten. This would suggest that responsible members of human-dog dyads avoid situations of risk. In contrast, $50.3 \%$ of owners who reported having had a dog suffer vehicular trauma also reported significantly more unspecified trauma. These owners may represent the human half of a dysfunctional dyad, where inappropriate husbandry choices may result in an increased experience with both unspecified trauma and vehicular trauma in their dog ownership history (Rohlf et al., 2010).

In this study, among owners who reported having had a dog that bit another animal they also reported significantly more experience with a dog that bit a person $(51.1 \%)$ and a dog that was bitten (34.8\%). It is interesting to note that the association between having had a dog that bites and a dog that was bitten is stronger within the group of owners that also reported experience with a least one dog that bit a person. This would seem to support the idea that individuals who have dog ownership histories that include various types of dog aggression (dog-dog and/or dog-human) represent a dysfunctional dyad (Tami et al., 2008; Cornelissen and Hopster, 2010). In such cases, owners may not understand the importance of dog training and socialization or even be aware that they are part of a potentially dysfunctional partnership. These individuals may believe that they are providing adequate dog care, and it is here that educational programs maybe the most effective (Lakestani and Donaldson, 2015; Schwebel et al., 2015). Through education, the owner can be made to understand how they are contributing to the problem within the dyad and given the tools to make relevant and lasting changes.

By asking owners to report on how many dogs, they had cared for up until the moment they filled out the questionnaire, the intention was to evaluate, albeit in a preliminary fashion, if experience with owning a larger number of dogs changed the type of dog health histories reported. It has been suggested that the more experience a person has with individual animals, the more knowledgeable they will be (Bennett and Rohlf, 2007; O'Connor et al., 2016). The results of this study are not in agreement with this statement, at least when it comes to dog on dog aggression. People who reported having owned more than 11 dogs also reported more experience with having had at least one dog that bit another animal. This could be because more experience with dog ownership may make owners more careless when it comes to intra-species aggression (Kubinyi et al., 2009). It is important to note, however, that this study did not identify how many dogs were being cared for simultaneously. As such, it could be that this raise in intra-species aggression results from situations of overcrowding (Tami et al., 2008) and is not related to owner experience. In this case, these owners may represent dysfunctional dyads, since there is a limit to how many dogs one individual can safely and legally care for (Assembleia da República, 2003).

It has been suggested that medically unjustified euthanasia can be seen by owners as a simple solution to their particular "problem pet" (Houpt et al., 2007; Coe et al., 2014), and as such, could be a marker of dysfunctional dyads. In this population, however, owner experience with euthanasia was not helpful in anticipating the existence of dysfunctional dyads. The absence of any association between euthanasia and any other variable under study could be due to a negative cultural view on euthanasia in general, since many individuals wish their pet to have a natural death in the family home. This can be true in both functional and dysfunctional dyads.

As the human-dog bond becomes increasingly relevant, the problem of dysfunctional human-dog dyads has been receiving increased attention. Such dyads represent a problem to society and place the individuals within the dyad, both human and canine, at risk (Lambert et al., 2015; Mongillo et al., 2015). As previously stated, human members of dysfunctional dyads are notoriously difficult to study, making these relationships visible only after the negative impact of inappropriate canine behavior has been felt (Drobatz and Smith, 2003; Kahn et al., 2003; Rosado et al., 2009; Matthias et al., 2015). This study has shown that it should be possible 
to identify potential characteristics both of functional and dysfunctional dyads by using data from a simple one page yes/no questionnaire. Human members of functional dyads tend to be responsible for their dogs' wellbeing, providing the necessary veterinary care and avoiding situations of risk. On the other hand, owners that maybe part of dysfunctional dyads show the opposite characteristics, reporting experience with various kinds of trauma and dog aggression. This suggests that human members of dysfunctional dyads may not be aware that their individual husbandry choices maybe placing themselves, and their dog, at risk.

More studies are required to understand whether these findings can be applied to other populations, namely, ones that are not urban in nature. Future studies should also endeavor to confirm the findings here described by validating the existence of such dog health histories in actual dysfunctional dyads. Preemptive identification of dysfunctional dyads would allow for the development of strategies and tools to limit the negative effect these dyads on each member and on society.

\section{Acknowledgments}

The authors would like to thank the various veterinary hospitals and clinics, as well as municipal veterinarians who helped make the gathering of data possible. Funding for this study was provided by CIISA-FMV ULisboa (Project UID/CVT/00276/2013, funded by FCT).

\section{Conflict of interest}

The authors declared that they have no conflict of interest.

\section{References}

Assembleia da República. 2003. Decreto Lei $\mathrm{n}^{\circ}$ 314/2003 de 17 de Dezembro. Diário da República $n^{\circ}$ 290/03 - I Série A. Ministérios das Finanças, da Administração Interna e da Agricultura, do Mar, do Ambiente e do Ordenamento do Território. Lisboa.

Bennett, P.C. and Rohlf, V.I. 2007. Owner-companion dog interactions: relationships between demographic variables, potentially problematic behaviours, training engagement and shared activities. Appl. Anim. Behav. Sci. 102(1-2), 65-84.

Bower, C. 2014. The complex issue of dog bites. Vet. Rec. 175,385 .

Le Brech, S., Amat, M., Camps, T., Temple, D. and Manteca, X. 2016. Canine aggression toward family members in Spain: clinical presentations and related factors. J. Vet. Behav. Clin. Appl. Res. 12, 36-41.

Coe, J.B., Young, I., Lambert, K., Dysart, L., Borden, L.N., Raji, A., Nogueira Borden, L. and Rajić, A. 2014. A scoping review of published research on the relinquishment of companion animals. J. Appl. Anim. Welf. Sci. 17(3), 253-273.

Cornelissen, J.M.R. and Hopster, H. 2010. Dog bites in The Netherlands: a study of victims, injuries, circumstances and aggressors to support evaluation of breed specific legislation. Vet. J. 186(3), 292-298.

Costa, P.S., Santos, N.C., Cunha, P., Cotter, J. and Sousa, N. 2013. The use of multiple correspondence analysis to explore associations between categories of qualitative variables in healthy ageing. J. Aging Res. e302163.

Davis, B.W., Nattrass, K., O'Brien, S., Patronek, G. and MacCollin, M. 2004. Assistance dog placement in the pediatric population: benefits, risks, and recommendations for future application. Anthrozoos 17(2), 130-145.

Diesel, G., Brodbelt, D. and Pfeiffer, D.U. 2010. Characteristics of relinquished dogs and their owners at 14 rehoming centers in the United Kingdom. J. Appl. Anim. Welf. Sci. 13(1), 15-30.

Drobatz, K.J. and Smith, G. 2003. Evaluation of risk factors for bite wounds in a veterinary teaching hospital. J. Am. Vet. Med. Assoc. 223(3), 312-316.

Fatjo, J., Amat, M., Mariotti, V.M., de la Torre, J.L.R. and Manteca, X. 2007. Analysis of 1040 cases of canine aggression in a referral practice in Spain. J. Vet. Behav. Clin. Appl. Res. 2(5), 158-165.

Galvis, J.O.A., Baquero, O.S., Dias, R.A., Ferreira, F., Chiozzotto, E.N. and Grisi. 2015. Monitoring techniques in the capture and adoption of dogs and cats. Geospat. Health 10(339), 158-162.

Greatbatch, I., Gosling, R.J. and Allen, S. 2015. Quantifying search dog effectiveness in a terrestrial search and rescue environment. Wilderness Environ. Med. 26(3), 327-334.

Guy, N.C., Luescher, U.A., Dohoo, S.E., Spangler, E., Miller, J.B., Dohoo, I.R. and Bate, L.A. 2001. A case series of biting dogs: characteristics of the dogs, their behaviour, and their victims. Appl. Anim. Behav. Sci. 74(1), 43-57.

Houpt, K.A., Goodwin, D., Uchida, Y., Baranyiová, E., Fatjó, J. and Kakuma, Y. 2007. Proceedings of a workshop to identify dog welfare issues in the US, Japan, Czech Republic, Spain and the UK. Appl. Anim. Behav. Sci. 106, 221-233.

Kahn, A., Bauche, P. and Lamoureux, J. 2003. Child victims of dog bites treated in emergency departments: a prospective survey. Eur. J. Pediatr. 162(4), 254-258.

Keuster, T.D., Lamoureux, J. and Kahn, A. 2006. Epidemiology of dog bites: a Belgian experience of canine behaviour and public health concerns. Vet. J. 172(3), 482-487.

Kuban, M., Królikowski, J. and Nowicki, M. 2016. Dog ownership status and self-assessed health, life-style and habitual physical activity in chronic hemodialysis patients. Hemodial. Int. 20(3), 447-452.

Kubinyi, E., Turcsán, B. and Miklósi, Á. 2009. Dog and owner demographic characteristics and dog personality trait associations. Behav. Processes 81(3), 392-401. 
Lakestani, N. and Donaldson, M.L. 2015. Dog bite prevention: Effect of a short educational intervention for preschool children. PLoS One 10(8), 1-14.

Lambert, K., Coe, J., Niel, L., Dewey, C. and Sargeant, J.M. 2015. A systematic review and meta-analysis of the proportion of dogs surrendered for dogrelated and owner-related reasons. Prev. Vet. Med. 118(1), 148-160.

Mariti, C., Gazzano, A., Moore, J.L., Baragli, P., Chelli, L. and Sighieri, C. 2012. Perception of dogs' stress by their owners. J. Vet. Behav. Clin. Appl. Res. 7(4), 213-219.

Matthias, J., Templin, M., Jordan, M.M. and Stanek, D. 2015. Cause, setting and ownership analysis of dog bites in Bay County, Florida from 2009 to 2010. Zoonoses Public Health 62(1), 38-43.

Meyer, I. and Forkman, B. 2014. Dog and owner characteristics affecting the dog-owner relationship. J. Vet. Behav. Clin. Appl. Res. 9(4), 143-150.

Mongillo, P., Adamelli, S., Pitteri, E. and Marinelli, L. 2015. Attention of dogs and owners in urban contexts: public perception and problematic behaviors. J. Vet. Behav. Clin. Appl. Res. 10(3), 210-216.

Mustiana, A., Toribio, J.A., Abdurrahman, M., Suadnya, I.W., Hernandez-Jover, M., Putra, A.A.G. and Ward, M.P. 2015. Owned and unowned dog population estimation, dog management and dog bites to inform rabies prevention and response on Lombok Island, Indonesia. PLoS One 10(5), 1-15.

Nimer, J. and Lundahl, B. 2007. Animal-assisted therapy : a meta-analysis. Anthrozoos 20(3), 225238.

O’Connor, R., Coe, J.B., Niel, L. and Jones-Bitton, A. 2016. Effect of adopters' lifestyles and animalcare knowledge on their expectations prior to companion-animal guardianship. J. Appl. Anim. Welf. Sci. 19(2), 157-170.

O'Haire, M. 2010. Companion animals and human health: benefits, challenges, and the road ahead. J. Vet. Behav. Clin. Appl. Res. 5(5), 226-234.

Overall, K.L. 2010. Breed specific legislation: How data can spare breeds and reduce dog bites. Vet. J. 186(3), 277-279.

Polzin, D.J. 2013. Evidence-based step-wise approach to managing chronic kidney disease in dogs and cats. J. Vet. Emerg. Crit. Care 23(2), 205-215.

Pouchelon, J.L., Atkins, C.E., Bussadori, C., Oyama, M.A., Vaden, S.L., Bonagura, J.D., Chetboul, V., Cowgill, L.D., Elliot, J., Francey, T., Grauer, G.F., Luis Fuentes, V., Sydney Moise, N., Polzin, D.J., Van Dongen, A.M. and Van Israël, N. 2015. Cardiovascular-renal axis disorders in the domestic dog and cat: a veterinary consensus statement. J. Small Anim. Pract. 56(9), 537-552.

Rehn, T. and Keeling, L.J. 2016. Measuring dog-owner relationships: crossing boundaries between animal behaviour and human psychology. Appl. Anim. Behav. Sci. 183, 1-9.

Robinson, N.J., Dean, R.S., Cobb, M. and Brennan, M.L. 2015. Investigating common clinical presentations in first opinion small animal consultations using direct observation. Vet. Rec. 176(18), 463.

Robinson, N.J., Dean, R.S., Cobb, M. and Brennan, M.L. 2016. Factors influencing common diagnoses made during first-opinion small-animal consultations in the United Kingdom. Prev. Vet. Med. 131, 87-94.

Rohlf, V.I., Bennett, P.C., Toukhasti, S. and Coleman, G. 2012. Beliefs underlying dog owners health care behaviors: results from a large, self-selected, Internet sample. Anthrozoos 25(2), 171-185.

Rohlf, V.I., Bennett, P.C., Toukhsati, S. and Coleman, G. 2010. Why do even committed dog owners fail to comply with some responsible ownership practices? Anthrozoos 23(2), 143-155.

Rosado, B., García-Belenguer, S., León, M. and Palacio, J. 2009. A comprehensive study of dog bites in Spain, 1995-2004. Vet. J. 179(3), 383-391.

Sanders, C.R. 2000. The impact of guide dogs on the identity of people with visual impairments. Anthrozoos 13(3), 131-139.

Schwebel, D.C., McClure, L.A. and Severson, J. 2015. Evalutaing a website to teach children safety with dogs. Inj. Prev. 21(1), 3279-3288.

Sterneberg-van der Maaten, T., Turner, D., Van Tilburg, J. and Vaarten, J. 2016. Benefits and risks for people and livestock of keeping companion animals: searching for a healthy balance. J. Comp. Pathol. 155(1), S8-S17.

Tami, G., Barone, A. and Diverio, S. 2008. Relationship between management factors and dog behavior in a sample of Argentine Dogos in Italy. J. Vet. Behav. Clin. Appl. Res. 3(2), 59-73.

Tavakol, M. and Dennick, R. 2011. Making sense of Cronbach's alpha. Int. J. Med. Educ. 2, 53-55.

Wang, G.-D., Zhai, W., Yang, H.-C., Wang, L., Zhong, L., Liu, Y.-H., Fan, R.-X., Yin, T.-T., Zhu, C.-L., Poyarkov, A.D., Irwin, D.M., Hytönen, M.K., Lohi, H., Wu, C.-I., Savolainen, P. and Zhang, Y.-P. 2015. Out of southern East Asia: the natural history of domestic dogs across the world. Cell Res. 26(1), $1-13$.

Wiseman-Orr, M.L., Nolan, A.M., Reid, J. and Scott, E.M. 2004. Development of a questionnaire to measure the effects of chronic pain on healthrelated quality of life in dogs. Am. J. Vet. Res. 65(8), 1077-1084.

Wood, L., Giles-Corti, B. and Bulsara, M. 2005. The pet connection: pets as a conduit for social capital? Soc. Sci. Med. 61(6), 1159-1173.

Yeates, J.W. and Main, D.C.J. 2011. Veterinary opinions on refusing euthanasia: justifications and philosophical frameworks. Vet. Rec. 168(10), 263. 